\title{
Sphincter of Oddi dysfunction produces acute pancreatitis in the possum
}

\author{
J W C Chen, A Thomas, C M Woods, A C Schloithe, J Toouli, G T P Saccone
}

\begin{abstract}
Background-Sphincter of Oddi dysfunction has been implicated as a cause of various forms of acute pancreatitis. However, there is no direct evidence to show that sphincter of Oddi dysfunction can cause obstruction of trans-sphincteric flow resulting in acute pancreatitis.

Aims-To determine if induced sphincter of Oddi spasm can produce transsphincteric obstruction and, in combination with stimulated pancreatic secretion, induce acute pancreatitis.

Methods-In anaesthetised possums, the pancreatic duct was ligated and pancreatic exocrine secretion stimulated by cholecystokinin octapeptide/secretin to induce acute pancreatitis. In separate animals, carbachol was applied topically to the sphincter of Oddi to cause transient sphincter obstruction. Sphincter of Oddi motility, trans-sphincteric flow, pancreatic duct pressure, pancreatic exocrine secretion, plasma amylase levels, and pancreatic tissue damage (histology score) were studied and compared with variables in ligation models.
\end{abstract}

Results-Acute pancreatitis developed following stimulation of pancreatic exocrine secretion with peptides after pancreatic duct ligation $(p<0.05)$. Neither pancreatic duct ligation nor stimulation of pancreatic exocrine secretion with cholecystokinin octapeptide/secretin alone resulted in acute pancreatitis. Topical carbachol stimulated sphincter of Oddi motility abolished trans-sphincteric flow, and increased pancreatic exocrine secretion $(p<0.05)$ and pancreatic duct pressure to levels comparable with pancreatic duct ligation $(p<0.001)$. Carbachol application (with or without combined peptide stimulation) elevated plasma amylase levels $(p<0.01)$ and produced pancreatic tissue damage $(p<0.05)$. Decompression of pancreatic duct ameliorated these effects $(p<0.05)$.

Conclusion-Induced sphincter of Oddi dysfunction when coupled with stimulated pancreatic secretion causes acute pancreatitis. This may be an important pathophysiological mechanism causing various forms of acute pancreatitis. (Gut 2000;47:539-545)

Keywords: acute pancreatitis; sphincter of Oddi; cholecystokinin octapeptide; possum
Sphincter of Oddi (SO) dysfunction has been implicated as a possible mechanism in some forms of acute pancreatitis. ${ }^{1} \mathrm{SO}$ dysfunction is defined as a manometric abnormality of the sphincter leading to impeded flow of bile or pancreatic juice. ${ }^{2}$ This abnormality has been shown to be associated with recurrent biliarytype pain ${ }^{3}$ and idiopathic recurrent pancreatitis. ${ }^{45}$ SO dysfunction has been implicated as a pathophysiological mechanism in other forms of acute pancreatitis such as gall stone pancreatitis, and pancreatitis secondary to alcohol, scorpion envenomation, organophosphate poisoning, and octreotide treatment. ${ }^{1}$ It has been postulated that transient or induced SO dysfunction due to oedema or spasm may be the primary mechanism leading to pancreatic duct obstruction in these cases.

The SO is under neurohormonal control. As cholinergic innervation of the $\mathrm{SO}$ is stimulatory, it has been postulated that acute pancreatitis resulting from excessive cholinergic stimulation with scorpion venom ${ }^{6}$ may be secondary to SO spasm in the presence of stimulated pancreatic exocrine secretion. Previous animal studies have suggested pancreatic duct obstruction at the level of $\mathrm{SO}^{78}$ during cholinergic hyperstimulation induced acute pancreatitis but there is no direct evidence of increased SO activity. Pancreatic exocrine secretion and pancreatic duct pressure increased in the presence of cholinergic stimulation ${ }^{9}$ but it is uncertain if these changes are secondary to induced $\mathrm{SO}$ dysfunction. Even if SO dysfunction contributes to increased pancreatic duct pressure, this may not be the primary cause of all forms of acute pancreatitis, as suggested by some studies. ${ }^{10} 11$

Australian Brush tailed possums (Trichosurus vulpecula) were used in this study because of their similarity to the human pancreatic and bile ducts arrangement in that they come together and are regulated by an SO complex. This SO complex is longer and predominantly extraduodenal allowing for easier manometric monitoring and topical pharmacological manipulation. The ducts drain through the same ampulla but there is no evidence of a common channel. Also, there is no evidence of an accessory duct (unlike the accessory duct of Santorini in humans), as assessed in over 200 possum pancreatic specimens. The comparative morphology of the SO between possums, humans, and other species has been described. ${ }^{12}$

The aims of the present study were to determine if (1) fixed obstruction by pancreatic duct

Abbreviations used in this paper: $\mathrm{SO}$, sphincter of Oddi; CCK-8, cholecystokinin octapeptide. 
ligation with stimulated pancreatic exocrine secretion by cholecystokinin octapeptide (CCK-8)/secretin and (2) transient SO obstruction with topical carbachol and stimulated pancreatic exocrine secretion alter plasma amylase levels and induce acute pancreatitis.

Table 1 Experimental groups

\begin{tabular}{lll}
\hline Group & & $n$ \\
\hline & Fixed obstruction & 5 \\
A & Sham & 5 \\
B & Pancreatic duct ligation alone & 5 \\
C & Pancreatic duct ligation+secretin stimulation & 5 \\
D & Pancreatic duct ligation+CCK-8/secretin stimulation & 5 \\
E & Pancreatic duct decompression+CCK-8/secretin stimulation & 5 \\
& Transient SO obstruction & 5 \\
G & SO obstruction by carbachol stimulation & 5 \\
H & SO obstruction by carbachol stimulation, pancreatic duct decompressed & 5 \\
\hline
\end{tabular}

CCK-8, cholecystokinin octapeptide; SO, sphincter of Oddi.
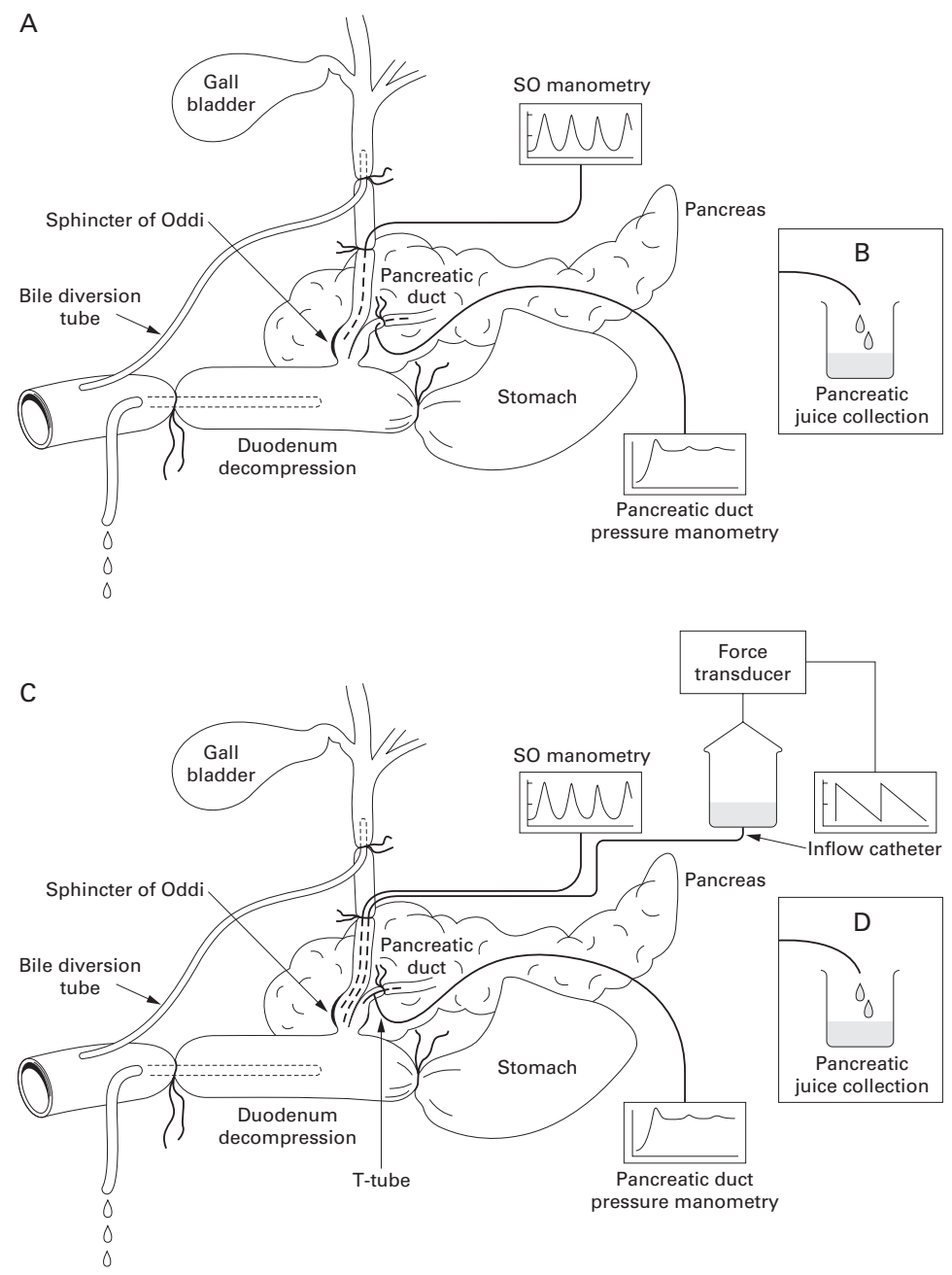

Figure 1 Schematic representation of the animal preparations used in this study. (A) Features of the preparations used for pancreatic duct ligation (groups $B, C$ and $D$ in table 1), showing bile diversion tube, sphincter of Oddi (SO) manometry catheter, duodenal decompression tube and pancreatic duct catheter for obstructive pressure measurement. (B) Modification incorporated for groups $A, E$, and $G$ which were subjected to pancreatic duct decompression. The pancreatic duct catheter was allowed to drain freely at $1 \mathrm{~cm}$ below the duodenum for pancreatic juice collection. Pancreatic amylase and volume were subsequently measured. (C) Key features for groups $F$ and $H$, which were prepared for topical carbachol stimulated SO obstruction. An inflow catheter was inserted into the common bile duct. Trans-sphincteric flow was measured by the rate of emptying of the reservoir containing saline. Partial/complete obstruction of the pancreatic duct was indicated by an increase in intraductal pressure, as measured through the T-tube in the pancreatic duct. (D)

Modification incorporated for group $G$ which had the pancreatic duct decompressed by a catheter (as shown in (B)).

\section{Methods}

ANIMAL PREPARATION

Forty Australian Brush tailed possums of both sexes $(1.5-3.5 \mathrm{~kg})$ were prepared as previously described. ${ }^{13} 14$

Briefly, anaesthesia was induced with a combination of intramuscular ketamine $(20 \mathrm{mg} / \mathrm{kg}$ Ketalar; Park Davis, Caringbah, NSW, Australia) and intramuscular xylazine $(10 \mathrm{mg} / \mathrm{kg}$, Rompun; Bayer, Botany, NSW, Australia). The left femoral vein was cannulated and anaesthesia was maintained for the duration of the experiment with a constant infusion of sodium pentobarbitone (Nembutal 15-20 $\mathrm{mg} / \mathrm{kg} / \mathrm{h}$; Boehringer Ingelheim Pty Ltd, Artarmon, NSW, Australia). Although ketamine has been shown to affect SO motility in some species, the effects, if any, on possum SO motility would have abated following surgical preparation of the animal (about one hour) and a stabilisation period of at least 15 minutes. The left femoral artery was cannulated and connected to a pressure transducer (Transpac IV, Abbot Ireland, Sligo, Republic of Ireland) for blood pressure monitoring. A tracheostomy was performed and the animal ventilated with a small animal ventilator (Phillips and Bird Inc., Richmond, Virginia, USA).

\section{Pancreatic duct ligation with stimulated}

pancreatic exocrine secretion studies (groups $A-E$ )

Five groups of animals $(\mathrm{n}=5)$ were subjected to pancreatic duct ligation or "pancreatic duct decompression" to characterise the effects of "fixed" pancreatic duct obstruction on the histological integrity of the pancreas and plasma levels of amylase (table 1). At laparotomy, a small choledochotomy was made in the common bile duct distal to the entry of the cystic duct. A single lumen side hole polyethylene manometry catheter (OD $1 \mathrm{~mm}$, ID $0.6 \mathrm{~mm}$ ) and a polyethylene bile diversion tube (OD 1.2 $\mathrm{mm}$, ID $0.8 \mathrm{~mm}$ ) were inserted (fig 1). ${ }^{13}{ }^{14}$ The manometry catheter, to measure SO motility, was connected to a minimally compliant pneumohydraulic pump (Arndorfer Medical Specialties Inc, Greenvale, Wisconsin, USA) and a pressure transducer. The pancreatic duct proximal to the SO was incised and one end of a polyethylene catheter (OD $1 \mathrm{~mm}$, ID 0.5 $\mathrm{mm}$ ) was inserted proximally $1-2 \mathrm{~mm}$ and secured with a 6.0 silk suture. In animals subjected to ligation of the pancreatic duct without decompression (groups B-D), the other end of this tube was connected to a pressure transducer with zero set at the level of the SO (fig $1 \mathrm{~A})$. In the pancreatic duct decompression groups (A, E), the end of the polyethylene catheter was set at a point about $1 \mathrm{~cm}$ lower than the duodenum to collect pancreatic juice for determination of pancreatic exocrine secretion volume and amylase secretion rate (fig 1B). All preparations were allowed to stabilise for at least 15 minutes prior to collection of juice or pancreatic duct ligation. Synthetic CCK-8 and secretin (porcine, Auspep, Parkville, Victoria, Australia) were dissolved in saline containing $0.01 \%$ bovine serum albumin (Sigma Chemical Co., St Louis, Missouri, USA) and administered as bolus injections 


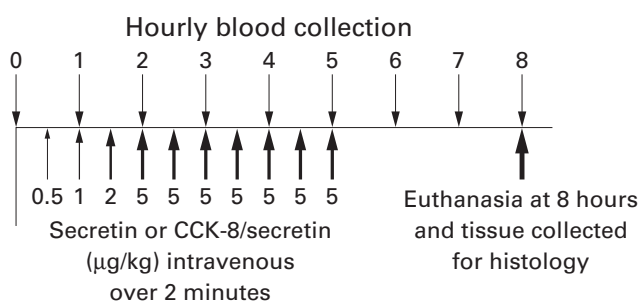

Figure 2 Experimental protocol for blood collection and peptide administration. Experiments were carried out over an eight hour period during which hourly plasma samples (small arrow, top) were taken for amylase measurement. In groups $C, D, E$, and $H$, which were subjected to secretin and or cholecystokinin octapeptide (CCK-8) stimulation, increasing intravenous bolus injection volumes (heavy arrow, bottom) were given within the first two hours, then doses of $5 \mu \mathrm{g} / \mathrm{kg}$ were administered at 30 minute intervals from two to five hours after ligation or start of the experiment. Eight hours after ligation, animals were killed and tissues collected for subsequent histological examination.

$(0.25-1.25 \mathrm{ml})$ via the femoral vein, at 30 minute intervals for a total of 10 injections over five hours. The initial dose $(0.5 \mu \mathrm{g} / \mathrm{kg}$ each $)$ of CCK-8/secretin was followed by increasing doses from 1 to $5 \mu \mathrm{g} / \mathrm{kg}$, and maintenance of this dose for a further three hours (fig 2). The final dose was given at five hours after pancreatic duct ligation.

Transient SO obstruction studies (groups $F-H$ ) An inflow catheter was inserted into the common bile duct together with the manometry catheter to measure trans-sphincteric flow, as previously reported (fig 1C). ${ }^{13-15}$ Briefly, a
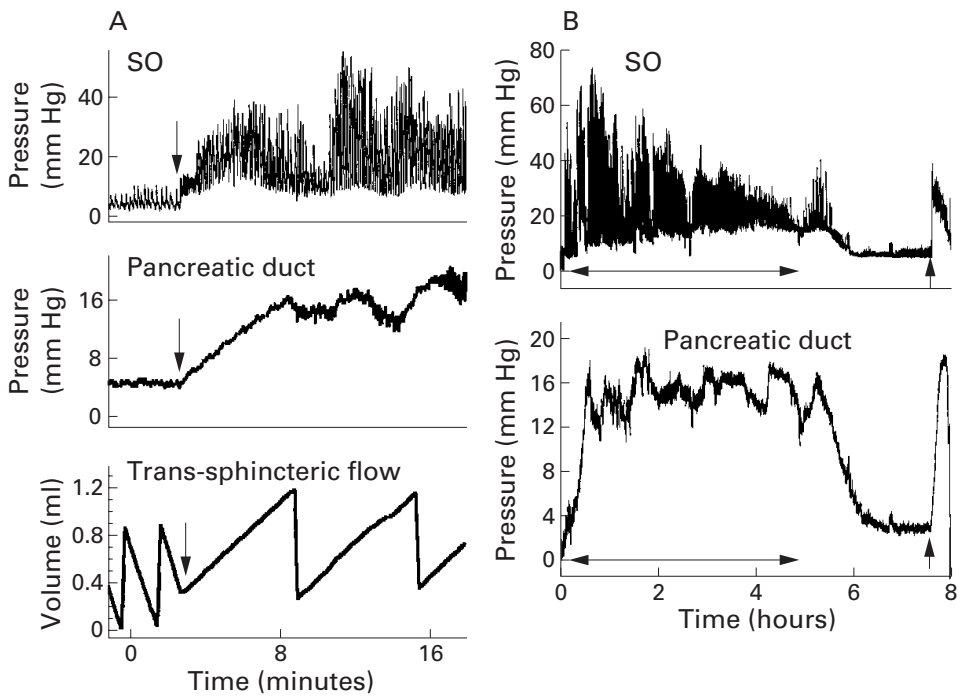

Figure 3 Effects of topical carbachol on sphincter of Oddi (SO) motility, pancreatic duct pressure, and trans-sphincteric flow. In $(A)$ and $(B)$, the vertical downward arrows indicate the beginning of topical carbachol administration. (A) Increased spontaneous $S O$ motility is seen following carbachol application. SO displays elevated basal pressure, phasic contraction frequency, and amplitude (top trace). This was associated with a corresponding increase in pancreatic duct pressure (middle trace) with concurrent cessation in trans-sphincteric flow (lower trace). The rapid upstrokes in the trans-sphincteric flow recordings prior to carbachol application represent rapid additions of $1 \mathrm{ml}$ of saline to the inflow reservoir to maintain a relatively constant inflow pressure. The period in which the flow rate changes from a negative to a positive slope indicates the period of cessation of trans-sphincteric flow. Cessation of trans-sphincteric flow was indicated as an upward slope as complete obstruction at the SO resulted in transfer of the manometry catheter perfusate into the saline reservoir via the inflow catheter. The rapid downstrokes (at about eight and 16 minutes) are due to aspiration of saline form the inflow reservoir to maintain a relatively constant inflow pressure. (B) Two channel recording over the duration (eight hours) of the experiments illustrating increased SO motility (upper trace) and elevated pancreatic duct pressure (lower trace) during the five hour period of topical carbachol application (horizontal double headed arrow). Towards the end of the experiment (7 hours 45 minutes), topical carbachol was reapplied (vertical arrow) to confirm the responsiveness of SO motility and pancreatic duct pressure. polyethylene inflow catheter (OD $1.2 \mathrm{~mm}$, ID $0.8 \mathrm{~mm}$ ) was positioned in the common bile duct proximal to the manometry catheter, secured with a ligature, and connected to a saline filled reservoir suspended from a force transducer (FT03, Grass Instruments, Quincy, Massachusetts, USA) acting as an electromagnetic balance. As trans-sphincteric flow proceeds, the weight of the reservoir decreases and the rate of change (slope) represents transsphincteric flow (fig 3A). Aliquots of saline (1 $\mathrm{ml}$ ) were delivered into the reservoir at regular intervals to maintain a relatively constant inflow pressure. Cessation of trans-sphincteric flow was indicated as an upward slope as complete obstruction at the SO resulted in transfer of the manometry catheter perfusate into the saline reservoir via the inflow catheter. Stimulation of SO activity to induce transient transsphincteric obstruction was achieved by multiple topical application $(2.5 \mu \mathrm{l})$ of carbachol (Sigma), initially $0.001 \mathrm{M}$, then at $0.01 \mathrm{M}$. Carbachol was applied topically using a pipette and placed directly onto the extraduodenal portion of the SO. Duration of topical carbachol induced cessation of transsphincteric flow through stimulation of SO was 1-10 minutes, with initial applications causing longer lasting effects. Cessation of transsphincteric flow was induced for a total of five hours (fig 3B). No additional carbachol was applied except for a test dose immediately prior to sacrifice of the animals at eight hours after induction of SO obstruction.

In groups $\mathrm{F}$ and $\mathrm{H}$, pancreatic duct pressure was measured for the duration of the experiment by insertion of a polyethylene T-tube catheter (OD $1.0 \mathrm{~mm}$, ID $0.5 \mathrm{~mm}$ ) into the pancreatic duct just proximal to the SO. This catheter was then connected to a saline filled pressure transducer (fig 1C). Group $\mathrm{H}$ was also subjected to CCK-8/secretin stimulation, as for groups D and E. In group G, carbachol was administered at the same concentrations used to stimulate cessation of trans-sphincteric flow, as indicated above. However, the pancreatic duct was decompressed by cannulation, as described above (fig 1D). All transducers were connected to a MacLab recording system (AD Instruments, Castle Hill, NSW, Australia). The possum's body temperature was maintained by a homeothermic warming blanket (Harvard Instruments, Boston, Massachusetts, USA). On completion of the experiment, all animals were killed with a lethal dose of sodium pentobarbitone (Lethabarb, Virbac, Peakhurst, NSW, Australia).

Blood pressure ( $\mathrm{mm} \mathrm{Hg}$ ), plasma amylase concentration (U/1), pancreatic amylase secretion $(\mathrm{U} / \mathrm{h})$, pancreatic exocrine volume secretion ( $\mathrm{ml} / 8 \mathrm{~h})$, SO manometry ( $\mathrm{mm} \mathrm{Hg}$ ), transsphincteric flow $(\mu \mathrm{l} / \mathrm{min})$, pancreatic duct pressure $(\mathrm{mm} \mathrm{Hg})$, and pancreas histology score (see below) were measured. The biliary portion of SO manometry was measured in all animals to confirm the presence of spontaneous activity throughout the studies and the effects of peptides and carbachol but SO activity was not quantified. The manometry of the pancreatic portion of SO was measured in pre- 
Table 2 Pancreatitis histology score

\begin{tabular}{ll}
\hline Histological feature & Score \\
\hline Oedema & $1-3$ \\
Fat inflammation & $1-3$ \\
Parenchymal inflammation & $1-3$ \\
Peripheral necrosis & $1-2$ \\
Fat necrosis & $3-7$ \\
Parenchymal necrosis & $3-7$ \\
\hline
\end{tabular}

Based on modification of Spormann et al. ${ }^{16}$

liminary studies and noted to have similar basal and phasic responses to high dose CCK-8, secretin, and carbachol as the biliary component. Therefore, only manometry of the biliary component was monitored in all animals.

PANCREATIC DUCT PRESSURE

Baseline pancreatic duct pressure was taken at the end of the stabilisation period. For possums subjected to pancreatic duct ligation, ligation pressure was taken as the peak pressure within one hour of ligation. For possums subjected to carbachol stimulated SO obstruction, the pancreatic duct obstructive pressure was taken as the peak pressure within one hour after induction of pancreatic duct obstruction.

Plasma amylase and pancreatic juice collection In all animals, $1 \mathrm{ml}$ of arterial blood was collected prior to laparotomy as a baseline measurement, at the beginning of induction of pancreatitis (time 0 ; fig 2), and then hourly for plasma amylase analysis. Blood samples were collected in EDTA tubes, centrifuged at $2000 \mathrm{rpm}$ for 10 minutes, and plasma stored at $-20^{\circ} \mathrm{C}$ overnight. For experiments with pancreatic duct decompression, after a 30 minute stabilisation period, pancreatic juices were collected hourly, with the secretion rate determined gravimetrically. Plasma and pancreatic juice amylase activity was measured using enzymatic colorimetric spectroanalysis (Boehringer Mannheim, Meylan, France) in a centrifugal analyser (Cobas Bios, F Hoffmann-La Roche, Basle, Switzerland). Plasma amylase levels were expressed as a percentage of baseline levels.

\section{Histological analysis}

The pancreas of each animal was harvested at eight hours, immediately fixed in $10 \%$ buffered formalin for 24-48 hours, and the head, neck, body and tail separated by dissection. Following paraffin embedding, sections were stained with haematoxylin-eosin and examined by a single pathologist blinded to the experimental protocol. A histology score was generated based on a modification of Spormann's criteria. ${ }^{16}$ The histological features assessed are listed in table 2 .

\section{STATISTICAL ANALYSIS}

Changes in plasma amylase levels were analysed using repeated measures analysis of variance (ANOVA). Changes in pancreatic secretory rate, amylase output, histology scores, and pancreatic duct pressure were analysed using one way ANOVA. A $p$ value $<0.05$ was regarded as significant. All data are expressed as mean (SEM) unless otherwise stated. The study was approved by the Animal Welfare Committee of Flinders University.

\section{Results}

PANCREATIC DUCT PRESSURE

Pancreatic duct pressure in groups B, C, and D with a catheter in situ were $4.2(0.7), 2.6(0.6)$, and $4.4(0.8) \mathrm{mm} \mathrm{Hg}$, respectively. These pressures increased to a mean of 17.8 (1.3), 18.0 (1.2), and $16.0(1.2) \mathrm{mm} \mathrm{Hg}$, respectively $(\mathrm{p}<0.001)$, within one hour of injection. Topical carbachol application induced a sustained increase in SO motility, with pancreatic duct pressure associated with cessation of transsphincteric flow (fig 3). Pancreatic duct pressure (prior to carbachol stimulated SO obstruction) was $5.6(0.5)$ and 4.3 (0.7) $\mathrm{mm} \mathrm{Hg}$ in groups $\mathrm{F}$ and $\mathrm{H}$, respectively. Following topical carbachol administration to achieve cessation of trans-sphincteric flow, pancreatic duct pressure in the nondecompressed groups (groups $\mathrm{F}$ and $\mathrm{H}$ ) increased to a peak of $18.4(0.7)$ and 21.0 (1.1) $\mathrm{mm} \mathrm{Hg}$, respectively. The increase in pancreatic duct pressure was comparable with that of pancreatic duct ligation above.

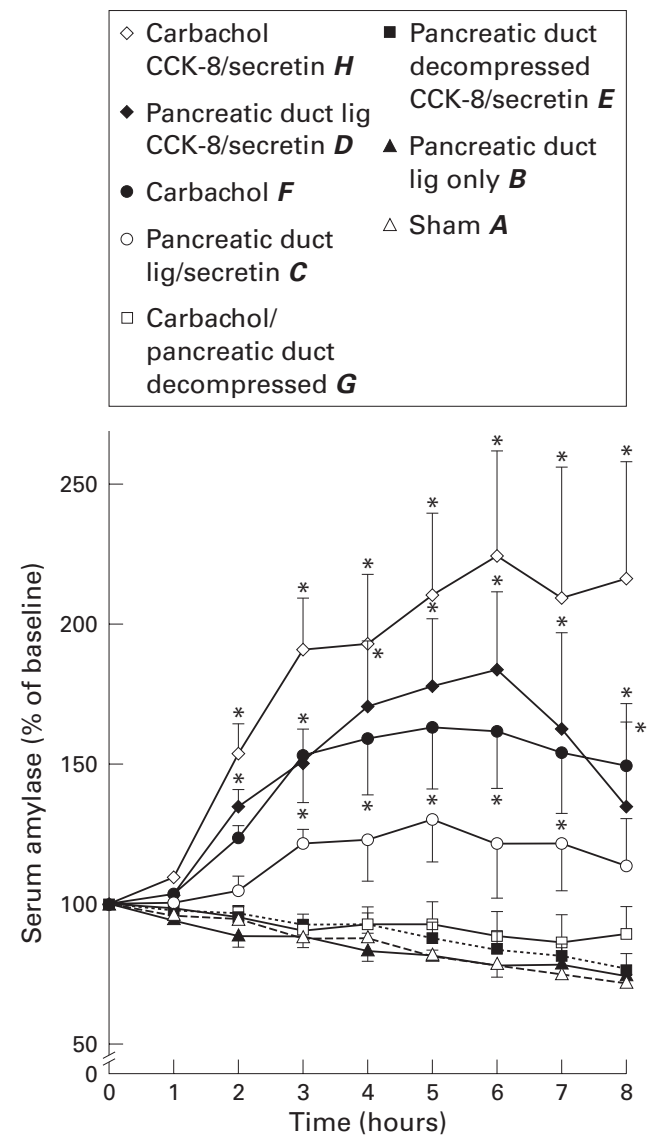

Figure 4 Plasma amylase profile (\% of baseline) of various experimental groups (indicated by bold italics) as a function of time. Significant increases in plasma amylase were noted in groups $D, F$, and $H$ compared with the sham (A), pancreatic duct ligation only (B), pancreatic duct decompressed (E), and carbachol administration with pancreatic duct decompression $(G)$ groups. ${ }^{\star} p<0.05$ compared with the sham group (repeated measures ANOVA). Data are mean (SEM) ( $n=5$ animals per group). 

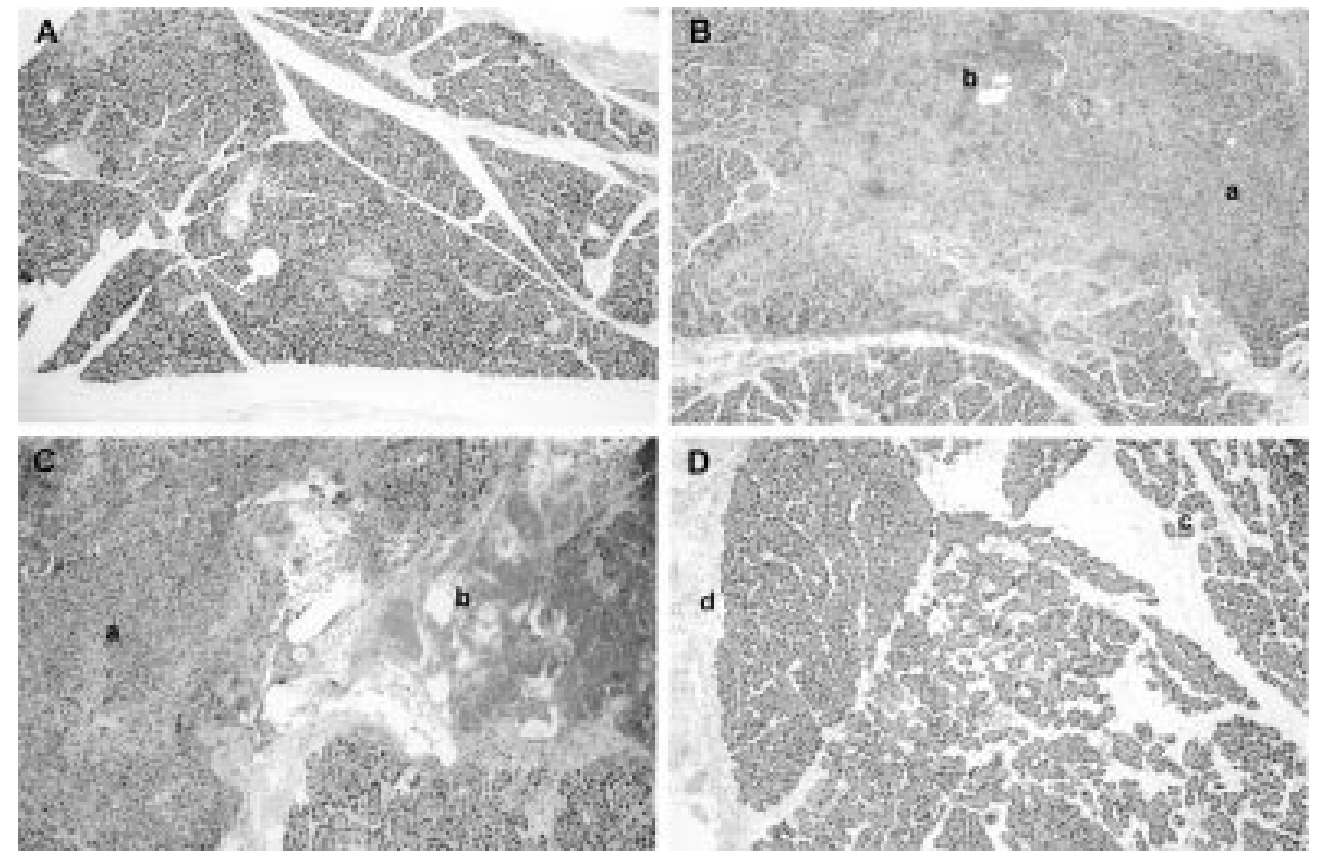

Figure 5 Representative histology slides of haematoxylin-eosin stained sections of possum pancreas from the sham group $(A)$, pancreatic duct ligation with secretin/CCK stimulation group (B), carbachol induced acute pancreatitis group (C) and carbachol induced SO obstruction in combination with secretin/CCK stimulation group (D). Minimal change is noted in (A) (sham). In (B) and (C), destruction of pancreatic acini with necrosis and inflammatory infiltration (a) is noted. Haemorrhage in these sections is also noted (b). In (D), diffuse parenchymal oedema (c) is noted with mild peripheral inflammatory infiltrate (d). (Original magnification $\times 25$.)

PANCREATIC EXOCRINE VOLUME AND AMYLASE SECRETION

Administration of CCK-8/secretin without pancreatic duct ligation (group E) significantly increased $(\mathrm{p}<0.02)$ pancreatic exocrine amylase secretion (496.3 (109.3) U) compared with the sham group (39.4 (17.4) U), but pancreatic secretion volume $(5.6(1.4) \mathrm{ml}$ for group $\mathrm{E} v 2.1(0.5) \mathrm{ml}$ for sham) was not significantly changed. Topical carbachol however, significantly increased $(\mathrm{p}<0.05)$ pancreatic exocrine secretion volume $(7.9(2.0) \mathrm{ml})$ compared with the sham group $(2.1(0.5) \mathrm{ml})$ without significantly increasing pancreatic amylase secretion (241.4 (110.6) U for carba-

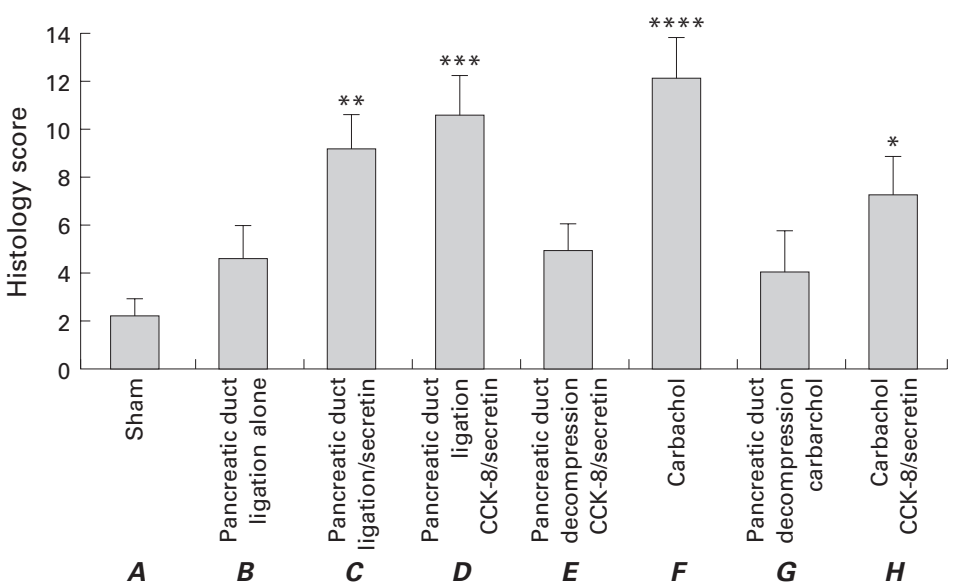

Figure 6 Pancreatitis histology score of various groups of animals (bold, italics, as defined in table 1). Acute pancreatitis histology scores were significantly increased in animals subjected to pancreatic duct obstruction with either secretin (group C), or secretin with cholecystokinin octapeptide (CCK-8) administration (group D), in animals subjected to carbachol stimulated transient sphincter of Oddi obstruction alone (group $F$ ) or with $C C K-8 /$ secretin administration (group $H$ ). Data are mean (SEM) ( $n=5$ animals per group). ${ }^{\star} p<0.02$ compared with the sham group (group $A$ ); ${ }^{\star *} p<0.05,{ }^{\star}{ }^{\star *} p<0.01$, $\star \star \star \star x<0.02$ compared with groups $A, B, E$, and $G$ (ANOVA). chol stimulated groups $v 39.4$ (12.4) U for the sham group).

\section{PLASMA AMYLASE}

Plasma amylase gradually decreased over the course of the experiment in the sham group (A), pancreatic duct ligation alone group (B), and CCK-8/secretin stimulation with pancreatic duct decompression group (E) (fig 4). Pancreatic duct ligation and simultaneous administration of CCK-8/secretin (group D) however resulted in significant hyperamylasaemia compared with the sham as well as the pancreatic duct decompressed group (E) $(p<0.005)$. Possums subjected to carbachol stimulated $\mathrm{SO}$ obstruction (groups $\mathrm{F}$ and $\mathrm{H}$ ) displayed a significant increase in plasma amylase levels $(p<0.01)$ which peaked at $5-6$ hours from the onset of carbachol administration (fig 4). Additional stimulation with CCK-8/ secretin (group $\mathrm{H}$ ) failed to significantly increase plasma amylase levels compared with the carbachol alone group $F(p=0.06)$. When the pancreatic duct was decompressed (group G), plasma amylase levels were comparable with those in the sham group.

ACUTE PANCREATITIS HISTOLOGY

Ligation of the pancreatic duct with secretin stimulation alone (group C) or with a combination of CCK-8 (group D) induced changes which included parenchymal oedema, and focal peripheral and central parenchymal fat necrosis (fig 5) resulting in a significantly increased histology score $(\mathrm{p}<0.05)$ compared with the sham group (fig 6). Mild changes with local oedema and inflammatory changes were noted in the sham group (A) which was subjected to the same degree of surgical 
dissection and handling as the other groups. The histology scores for pancreatic duct ligation alone (group B) and pancreatic duct decompression with secretin/CCK-8 stimulation (group E) were not significantly different from the sham histology score (fig 6). Carbachol induced transient SO obstruction with or without CCK-8/secretin stimulation (groups F and $\mathrm{H}$ ) resulted in significantly increased pancreatic damage - that is, higher histology score $(\mathrm{p}<0.001$ and $\mathrm{p}<0.02$, respectively) compared with the sham group (fig 4). Decompression of the pancreatic duct despite carbachol stimulated SO obstruction (group G) did not result in significant histological changes. Histology scores for the ligation and CCK-8/secretin stimulation group (D) were comparable with those of the transient SO obstruction by carbachol group (F) (fig 6).

\section{Discussion}

For the first time, we have shown that transient obstruction of the SO produced by carbachol administration resulted in hyperamylasaemia and histological changes consistent with acute pancreatitis. These changes were comparable with those induced in possums with combined pancreatic duct ligation and CCK-8/secretin administration, a well established method on inducing hyperamylasaemia and acute pancreatitis in other species. In both the carbachol induced and pancreatic duct ligation with secretin/CCK-8 models, the concurrent increase in pancreatic duct pressure with a combination of pancreatic exocrine stimulation was necessary to produce hyperamylasaemia and acute pancreatitis.

The increase in pressure in the pancreatic duct was similar whether it followed pancreatic duct ligation or was induced by carbachol administration. The increase in plasma amylase in all cases corresponded with the increase in pancreatic duct pressure following pancreatic duct ligation or stimulated SO obstruction in combination with pancreatic exocrine stimulation. When the pancreatic duct was decompressed by a polyethylene tube in the duct in these groups, plasma amylase decreased over the duration of the experiment. These findings indicate that pancreatic duct obstruction resulting in an increase in pancreatic duct pressure in combination with pancreatic exocrine stimulation is required to produce hyperamylasaemia and not pancreatic exocrine stimulation alone. It is not surprising to find that the combination of carbachol induced SO obstruction in the presence of CCK-8/secretin stimulation resulted in the highest increase in plasma amylase levels suggesting that secretion against an obstructed pancreatic duct is the cause of hyperamylasaemia in these cases. Although topical application of carbachol also stimulated exocrine pancreatic secretion volume, this did not result in a significant rise in plasma amylase or histology score when the pancreatic duct was decompressed, indicating that SO stimulated pancreatic duct obstruction coupling is essential in causing acute pancreatitis.
Pancreatic dissection and handling resulted in localised mild histological changes. When the animals were subjected to pancreatic duct ligation and CCK-8/secretin stimulation, a significant increase in pancreatitis histology score was noted. The pancreatitis occurred mainly in the head of the pancreas and was associated with diffuse oedema, peripheral necrosis of 1-2 cells thick, as well as areas of parenchymal necrosis. Similar changes were also noted in groups subjected to carbachol stimulated $\mathrm{SO}$ obstruction with or without additional peptide stimulation. This pancreatitis in possums is different from that noted after cerulein administration in the rat where high serum amylase levels associated with mainly pancreatic oedema is reported. ${ }^{17}$ The severity of acute pancreatitis in possums following pancreatic duct occlusion is greater than that produced in mice $^{18}$ following pancreatic duct ligation but less severe compared with ligation of the biliopancreatic duct of the opossum. ${ }^{19}$

In this study, possums were used as the motility of the SO has been well characterised. The $\mathrm{SO}$ of the possum acts as a variable resistor in modulating bile and pancreatic secretion and its responses to hormonal and drug stimuli are very similar to humans. ${ }^{13-1520}$ Possums have not been used previously in the study of acute pancreatitis and consequently pancreatic duct ligation studies were performed. Previous studies have shown that pancreatic duct obstruction coupled with pancreatic exocrine stimulation in animal models can result in hyperamylasaemia with oedematous pancreatitis. ${ }^{22}$ The severity of pancreatitis varies in different species using similar stimuli, ${ }^{23-26}$ but also varies with different stimuli used to produce pancreatitis. Our study showed that mild to moderate pancreatitis was produced by SO obstruction combined with increased pancreatic exocrine secretion. Furthermore, carbachol induced acute pancreatitis was similar to acute pancreatitis which may be produced following idiopathic SO dysfunction, or SO dysfunction following passage of a gall stone through the SO in biliary pancreatitis.

SO dysfunction has been implicated as a possible cause of various forms of acute pancreatitis, including idiopathic recurrent pancreatitis, gall stone pancreatitis, as well as pancreatitis due to scorpion toxin envenomation and drugs such as octreotide. ${ }^{1}$ Although it has been postulated that SO dysfunction either in the form of stenosis (oedema or SO hypertrophy) or dyskinesia (tachyoddia, induced spasm) leads to partial or complete obstruction of the pancreatic duct resulting in pancreatitis, this mechanism has not been demonstrated directly. Our results in possum strongly implicate SO dysfunction as one of the causative factors for production of pancreatitis. In particular, the changes in SO motility produced by carbachol clearly indicates that together with increased secretion by the pancreas, this is an essential combination in the production of this form of pancreatitis.

We have demonstrated that the $\mathrm{SO}$, which is strategically placed to modulate pancreatic and bile flow, can cause pancreatitis when stimulated to contract during periods of elevated 
pancreatic exocrine secretion. Therefore, simultaneous SO dysfunction (impeding flow) combined with elevated pancreatic exocrine secretion may be an important pathophysiological mechanism underlying various forms of acute pancreatitis. This newly described possum model will also enable the study of the role of SO motility in other forms of acute pancreatitis where SO dysfunction has been implicated.

The study was supported by the Royal Australasian College of Surgeons and the National Health and Research Council of Australia. Permission for the use of the Australian Brush tailed possum in this study was granted by the South Australian National Parks and Wildlife Service. The technical assistance of Mr A Citti is acknowledged.

1 Chen JW, Saccone GT, Toouli J. Sphincter of Oddi dysfunction and acute pancreatitis. Gut 1998;43:305-8.

2 Hogan WJ, Sherman S, Pasricha P, et al. Sphincter of Oddi manometry. Gastrointest Endosc 1997;45:342-8.

3 Toouli J, Roberts-Thompson I, Dent J, et al. Manometric disorders in patients with suspected sphincter of Oddi dysdisorders in patients with suspected sphincter
function. Gastroenterology 1985;88:1243-50.

4 Toouli J, Roberts-Thompson I, Dent J, et al. Sphincter of Toouli J, Roberts-Thompson I, Dent J, et al. Sphincter of
Oddi motility disorders in patients with idiopathic Oddi motility disorders in patients with idio
recurrent pancreatitis. Br f Surg 1985;72:859-63.

5 Toouli J, Di Francesco V, Saccone G, et al. Division of the sphincter of Oddi for treatment of dysfunction associated with recurrent pancreatitis. Br F Surg 1996;83:1205-10.

6 Novaes G, Catanzaro OL, Beraldo WT, et al. Effect of purified scorpion toxin (tityustoxin) on the pancreatic secretion of the rat. Toxicon 1982;20:847-53.

7 Bartholomew C, McGeeney KF, Murphy JJ, et al. Experimental studies on the aetiology of acute scorpion pancreatitis. Br F Surg 1976;63:807-10.

8 Novaes G, Cabral AP, de Falco CN, et al. Acute pancreatitis induced by scorpion toxin, tityustoxin. Histopathological study in rats. Arq Gastroenterol 1989;26:9-12.

9 Dressel TD, Goodale-RL J, Arneson MA, et al. Pancreatitis as a complication of anticholinesterase insecticide intoxicaas a complication of anticholinesteras
tion. Ann Surg 1979;189:199-204.

10 Dressel TD, Goodale-RL J, Zweber B, et al. The effect of atropine and duct decompression on the evolution of atropine and duct decompression on the evolution of Diazinon-induce

11 Pantoja JL, Renner IG, Abramson SB, et al. Production of acute hemorrhagic pancreatitis in the dog using venom of the scorpion, Buthus quinquestriatus. Dig Dis Sci 1983;28: 429-39.
12 Toouli J, Baker RA. Innervation of the sphincter of Oddi: physiology and considerations of pharmacological intervention in biliary dyskinesia. Pharmacol Ther 1991;49:26981.

13 Liu YF, Saccone GT, Thune A, et al. Sphincter of Oddi regulates flow by acting as a variable resistor to flow. $A m \mathcal{F}$ Physiol 1992;263:G683-9.

14 Saccone GT, Liu YF, Thune A, et al. Erythromycin and motilin stimulate sphincter of Oddi motility and inhibit trans-sphincteric flow in the Australian possum. Naunyn Schmiedebergs Arch Pharmacol 1992;346:701-6.

15 Baker RA, Wilson TG, Padbury RT, et al. Galanin modulates sphincter of Oddi function in the Australian brush-tailed possum. Peptides 1996;17:933-41.

16 Spormann H, Sokolowski A, Letko G. Experimental acute pancreatitis - a quantification of dynamics at enzymic and histomorphologic levels. Pathol Res Pract 1989;185:358-62.

17 Lampel M, Kern HF. Acute interstitial pancreatitis in the rat induced by excessive doses of a pancreatic secretagogue. Virchows Arch A Pathol Pathol Anat 1977;373:97-117.

18 Watanabe S, Abe K, Anbo Y, et al. Changes in the mouse exocrine pancreas after pancreatic duct ligation: a qualitative and quantitative histological study. Arch Histol Cytol 1995;58:365-74.

19 Lerch MM, Saluja AK, Dawra, et al. Acute necrotizing pancreatitis in the opossum: earliest morphological changes involve acinar cells. Gastroenterology 1992;103:205-13.

20 Toouli J, Baker RA. Innervation of the sphincter of Oddi: physiology and considerations of pharmacological intervention in biliary dyskinesia. Pharmacol Ther 1991;49:26981

21 Dumont AE, Martelli AB. Pathogenesis of pancreatic edema following exocrine duct obstruction. Ann Surg 1968;168: 302-9.

22 Churg A, Richter WR. Early changes in the exocrine pancreas of the dog and rat after ligation of the pancreatic duct. A light and electron microscopic study. Am F Pathol 1971;63:521-46.

23 Ohshio G, Saluja AK, Leli U, et al. Esterase inhibitors prevent lysosomal enzyme redistribution in two noninvasive models of experimental pancreatitis. Gastroenterology 1989; 96:853-9.

24 Foitzik T, Lewandrowski KB, Fernandez-del CC, et al. Exocrine hyperstimulation but not pancreatic duct obstruction increases the susceptibility to alcohol-related pancreatic injury. Arch Surg 1994;129:1081-5.

25 Luthen R, Niederau C, Niederau M, et al. Influence of ductal pressure and infusates on activity and subcellular distribution of lysosomal enzymes in the rat pancreas. Gastroenterology 1995;109:573-81.

26 Cavuoti OP, Moody FG, Martinez G. Role of pancreatic duct occlusion with prolamine (Ethibloc) in necrotizing pancreatitis. Surgery 1988;103:361-6. 